\title{
Characterization of Measurement Errors in a LBL Positioning System
}

\author{
Rui Almeida \\ FEUP \\ and INESC TEC \\ rui.almeida@fe.up.pt
}

\author{
José Melo \\ FEUP \\ and INESC TEC \\ jose.melo@fe.up.pt
}

\author{
Nuno Cruz \\ FEUP \\ and INESC TEC \\ nacruz@fe.up.pt
}

\begin{abstract}
There are several sources of error affecting the accuracy of underwater ranging using acoustic signals. These errors have a direct impact in the performance of Long Baseline (LBL) navigation system. This paper presents the results of experiments designed to characterize the most significant sources of errors in acoustic ranging. For the experiments, we use a set of acoustic devices and compare distances given by GPS differences with and acoustic ranges. We describe the experimental procedure and we process the results to provide a qualitative and quantitative analysis of the errors.
\end{abstract}

\section{INTRODUCTION}

Acoustic Navigation embraces a number of techniques that rely on the exchange of acoustic signals between a vehicle, which the position needs to be derived, and a set of existing acoustic beacons. Broadly speaking, three distinct Acoustic Navigation schemes exist, namely the Long Baseline (LBL), the Short Baseline (SBL), and the Ultra Short Baseline (USBL). Comparing to their counterparts, one of their main advantages of LBL systems is that they grant navigation capabilities in a wide area and have very good, depth independent, position accuracy, which falls in the meter scale. Because of that, LBL positioning systems have always played a major role in the field of underwater robotics. In this article, we will be focused exclusively on those.

LBL systems need to have an array of acoustic beacons deployed on the seafloor, in a predefined location within the operation area. The cost and time needed to set up and deploy the LBL network, and the later recovery of the beacons, can be quite cumbersome particularly in adverse environments. In fact, this can be considered one of the main drawbacks in the use of such systems.To overcome the need of deploying the beacons on the seafloor, the use of GPS-enabled buoys was proposed, in a configuration called Inverted LBL. With the use of such systems, the transponders of the bottom are replaced by floating buoys which carry the acoustic transducers. Due to the fact that such devices also carry GPS, calibration of the system can be significantly simplified. For a review of the different Acoustic Navigation schemes, their individual strengths and their disadvantages, refer to [1], [2].

The relative position of an Unmanned Underwater Vehicle (UUV) can be computed with an LBL system by acoustically measuring its distance to each beacon. This acoustic distance, or range, can be obtained by precisely measuring the Time of

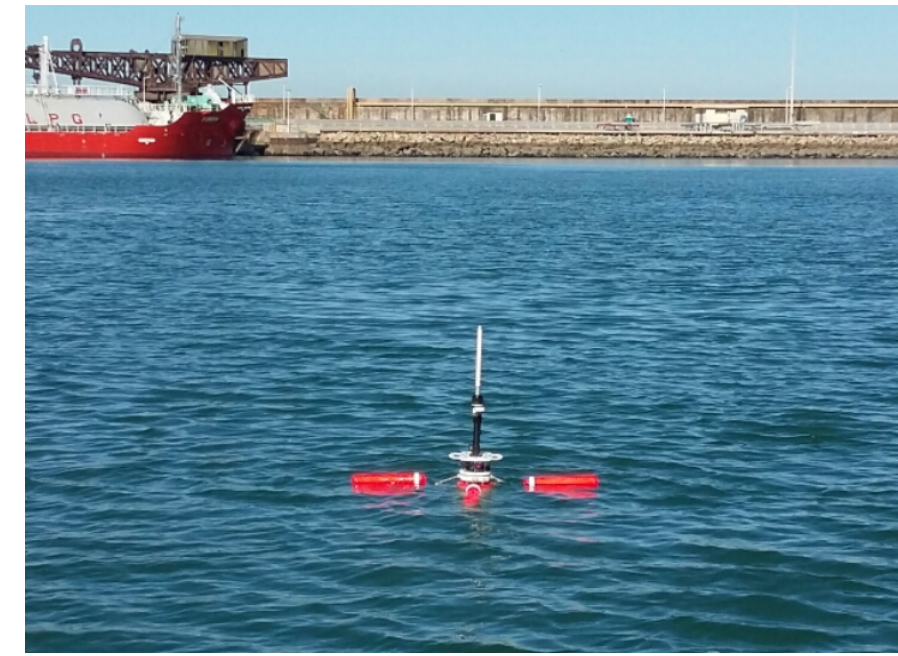

Fig. 1. Man Portable Acoustic Navigation Buoy deployed in Leixões harbor, Porto, Portugal

Flight (ToF) of the signals exchanged between the UUV and each of the beacons. However, these measurements are often affected by both system and environment dependent sources of error that can negatively affect the range measurements. Other authors have focused on the theoretical influence some of these errors can have on the accuracy of an LBL-derived position solution [3], [4]. In this article, the focus is on identify and experimentally characterize these sources of error. This is in fact relevant, as it can have a paramount influence in the maximum achievable positioning precision and accuracy of an LBL system.

The remaining of this article is organized as follows. In Section II we will detail the different methods for LBL Navigation, and in Section III we describe the hardware configuration used throughout this article. In Section IV we identify the different errors affecting the range measurements, and Section $V$ details the procedure to characterize these error sources. Finally, in Section VI we present the results from our experimental validation, and lastly, in Section VII we present some concluding remarks and point out future research directions. 


\section{LBL NAVIGATION SYSTEMS}

LBL Navigation Systems can provide relative positioning solutions of an UUV, whether for navigation or tracking, by acoustically measuring distances from the vehicle to a set of acoustic beacons, deployed in predefined positions. Traditionally this process is based on a interrogation protocol, on which the vehicle interrogates each one of the beacons, sending an acoustic signal, and waiting a response. After detecting this signal, the beacons are then supposed to reply, sending an acoustic signal back to the vehicle. Because of that, such LBL configurations are also known as Two-WayTravel-Time (TWTT). For practical matters, the reply signal by the beacon is usually only sent after a system dependent turn-around delay time. The measured ToF, the elapsed time between emission and reception, is then converted to the corresponding range measurement, or distance, by taking into account the speed of sound. An LBL based navigation and tracking application can be seen for example in [5].

The nature of the TWTT interrogation process doesn't require any kind of synchronism between the all the devices. This was an obvious advantage on the early days of this technology, as achieving synchronism between clock sources was not a straightforward process. Nevertheless, this method is not easily scalable for situations dealing with multiple vehicles. For this reason, alternative LBL schemes have been derived.

Opposed to traditional TWTT LBL systems, One-WayTravel-Time (OWTT) LBL systems have been proposed in order to enable acoustic navigation for multiple vehicles. While other works concerning OWTT systems had already been proposed in the literature, one of the first and fully developed systems enabling synchronous-clock, OWTT navigation for multiple vehicles was presented in [6], to which other articles that followed. This approach requires that all the devices of the acoustic network, beacons and vehicles, need to have their clock sources synchronized. In this scheme the beacons, also in predefined known positions, synchronously broadcast acoustic signals at previously established time instants. These signals will then be received by all the vehicles. Since the clocks are synchronized, it is possible for the vehicles to compute the ranges to each of the beacons by using only the OWTT, the elapsed time between the emission instant, known in advance, and reception instant.

Initially, the requirement for a synchronized clock source between all the vehicles was pointed out as a big drawback in OWTT based methods. Achieving the necessary timing accuracy is something that only recently was possible to reach with relatively easiness. Nowadays, the ubiquity of advanced GPS receivers able to output highly stable PulsePer-Second (PPS) signals, and the availability of low-drift clock sources, make this requirement relatively straightforward to match. Moreover, while initially LBL systems based on OWTT techniques were restricted to navigation-only, solutions allowing only tracking [7] and simultaneous navigation and tracking [8] have already emerged in the literature.

\section{HARDWARE DESCRIPTION}

For this trial we used two similar systems to perform, respectively, as moored and moving acoustic beacons. The core hardware of the these acoustic module was the same for both of them, but the surrounding hardware and mechanical support differed slightly. The software stack running in any of our systems allows them to be controlled by any remote PC or control station. This means that any transmission schemes can be triggered by either a local or remote control software.

The moored beacon was a Man Portable Acoustic Navigation Beacon, described in [9], can be seen in Figure 1. This system is fitted with a low power embedded PC running a distribution of Linux, a GPS receiver with PPS output, WiFi Dongle for direct wide-band access to the system and a serial radio modem for long range communications. The entire system was powered by a pack of Li-Po batteries. On the other hand, the moving beacon, from now on referred to as pinger, was equipped with a low power embedded ARM PC, running the same distribution of Linux of the moored station. It also features a GPS receiver with PPS output and the same serial radio modem for long range communications. For WiFi communications the system is fitted with a Bullet Access Point that both provided WiFi connection to the system and served as a field access point. Synchronization of both systems was achieved using the GPS PPS signals.

These systems where equipped with an acoustic transducer and a set of our acoustic modules. This modules, developed at our labs at INESC TEC, feature a "Control Board", a "Transmission Board", and a "Reception Board". A photo of these acoustic modules can be seen in Figure 2.

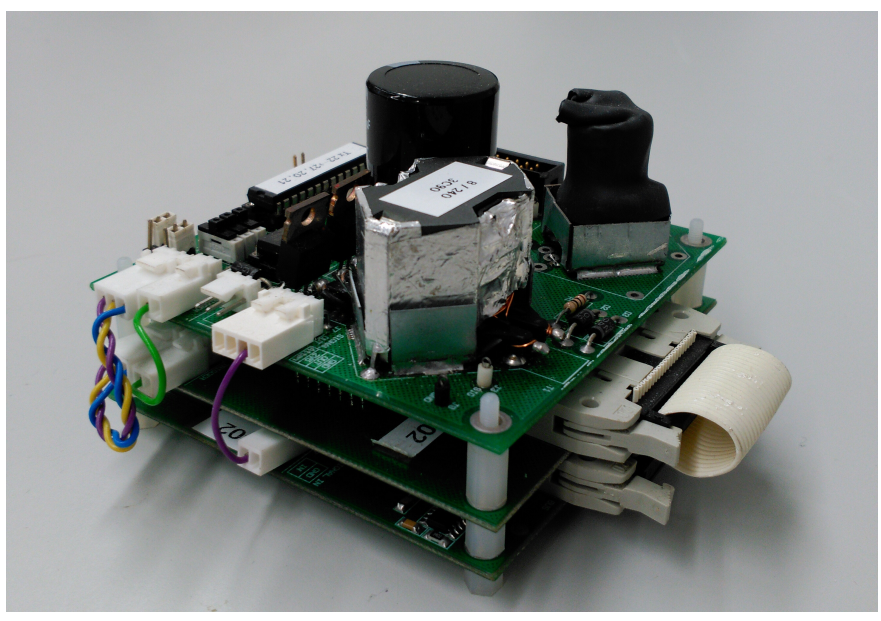

Fig. 2. Acoustic Modules

The "Control Board is responsible for handling the transmission and reception of the acoustic signals. The board features a microprocessor that measures the ToF of the acoustic signals with a timer/counter running at $10 \mathrm{kHz}$. Being synchronous with the PPS signal from the GPS receiver, the frequency of the counter imposes a quantization error of $15 \mathrm{~cm}$. The "Transmission Board", on top, houses the high power electronics 
that are capable of transmitting a sinusoidal wave in eight frequencies ranging from $20 \mathrm{kHz}$ to $27 \mathrm{kHz}$. Finally the "Reception Board", contains all the signal conditioning and filtering electronics required for detecting any one of the transmitted signals. This conditioning and filtering is made with analog electronics. They introduce a delay in the reception time of the acoustic signal, that needs to be quantified. This delay is dependent on both the power of the received signal and the environment noise.

\section{CharaCterization OF ERRORS}

As stated before the objective of this trial was to characterize the sources of error that affect the computation of acoustic ranges in underwater positioning or tracking systems. In LBL systems the position of a vehicle can be computed by acoustic ranging between the vehicle and each one of the beacons. Using traditional TWTT techniques, the range $d$ between the two beacons is computed by measuring the ToF, the elapsed time between the transmission of an acoustic signal and the reception of its response, and affect it by the speed of sound, $v_{s}$, as follows.

$$
d=\frac{T o F}{2} \cdot v_{s}
$$

Thus being simple, this computation (1) is prone to errors in timing, but also to errors introduced by the space and time varying nature of the sound of speed in the water column. Adding to it, signal acquisition induced delays, and the time resolution of the system lead to a decreased accuracy of the range measurements.

Timing errors can be divided into three types of sources, the difference in time of the clocks of the various systems, the drift of each clock and the uncertainty in the turn-around delay introduced by the microprocessor. Independently of the scheme used (OWTT or TWTT) our systems are synchronized with each other through the PPS signal of the GPS receivers. The PPS signal provided by our receivers was experimentally measured and we determined a maximum jitter of $25 \mathrm{~ns}$. This is equivalent to a maximum deviation of less than $1 \mathrm{~mm}$ in the computation of a range measurement. The microprocessor of the acoustic module has an inherent low drift and since it is reset every second, by the PPS signal, its drift is considered nonexistent for our system. When using a TWTT approach one must be aware of errors induced by the microprocessor when processing a reception and transmitting a reply after a predefined delay. The turn-around delay is a fixed delay that is built into the microprocessor and takes into account the processing time in order to produce a fixed response time to a reception. However since this delay is triggered in an interrupt base, in more extreme cases where the microprocessor is being more requested, a drift in the order of a microprocessor counter tic has been detected.

By construction the filters and conditioning electronics from the acoustic module take a certain amount of time to produce a detection since the reception of an acoustic signal on the acoustic transducer. This response time is dependent on power of the received acoustic signal. Theoretically this response time could be computed, if the power of the received signal was known. However the power of the signal is highly dependent on the environment conditions. Adding to it, even with very precise electronics the value of this response time can vary slightly from module to module, due to transducer characteristics. In the case of a OWTT scheme the response time can be experimentally measured for each of the receiving modules. On the other hand, with a TWTT approach we can only determine an average value of the response of the modules from the interrogator and the modules from the beacon that is replying.

Recalling equation 1, it does not take into account system imposed delays that might arise. In practice the elapsed time measured by LBL systems comes affected by different factors, as illustrated in Figure 3.

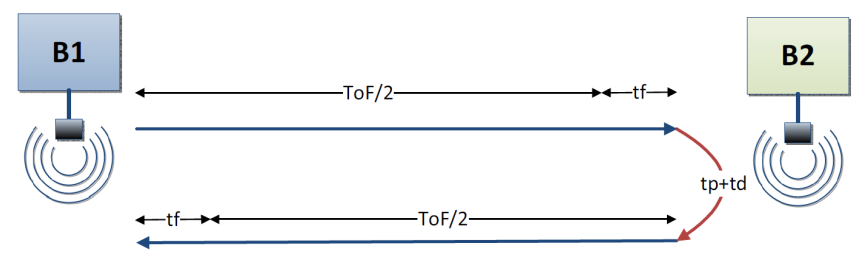

Fig. 3. Two Way Travel Time Signal Chain

The actual time measured by the system, $t_{A C S}$ is in fact a sum of different factors, for example delays introduced by electronic filters, $t_{f}$, processing times and other designspecified delays, $t_{p}+t_{d}$ :

$$
t_{A C S}=\frac{T o F}{2}+t_{f}+\left(t_{p}+t_{d}\right)+\frac{T o F}{2}+t_{f}
$$

Taking this in consideration, and substituting accordingly in (1), the actual range distance can then be obtained by:

$$
d_{T W T T}=\frac{t_{A C S}-2 t_{f}-\left(t_{p}+t_{d}\right)}{2} \cdot v_{s}
$$

A similar reasoning can be made for the OWTT case, illustrated in Figure 4. However, in this case the time measurements come only affected by $t_{f}$, the filtering induced delay.

$$
d_{O W T T}=\left(t_{A C S}-t_{f}\right) . v_{s}
$$

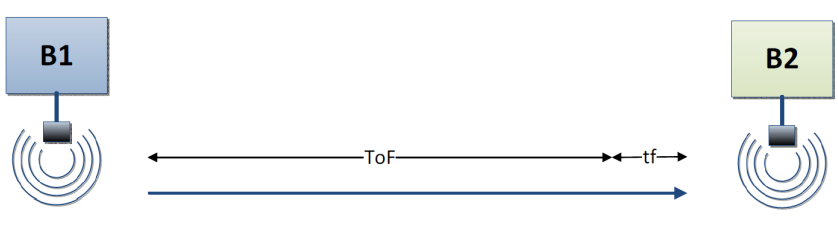

Fig. 4. One Way Travel Time Signal Chain

If we consider Equations 3 and 4 the main objective of this work is then to quantify $t_{f}$ and determine the average speed of sound for the operational scenario, as they can significantly 
affect the determination of an acoustic range. The procedure to determine these values is detailed in the next section.

\section{PROCEDURe}

In order to determine the sources of error in underwater acoustic ranging, we've designed a set of experiments, performed close to the Leixões harbor, in Porto, Portugal, in February 2016. To perform the experiments we used two acoustic systems, described before in Section III.

We've used two acoustic systems to perform these experiments. The man portable navigation beacon was moored throughout the whole experiments in about 8 meters of water, with the acoustic transducer located about $50 \mathrm{~cm}$ below the surface. The other system, the pinger, was transported in a RHIB and anchored in three different locations, as can be seen in Figure 5.

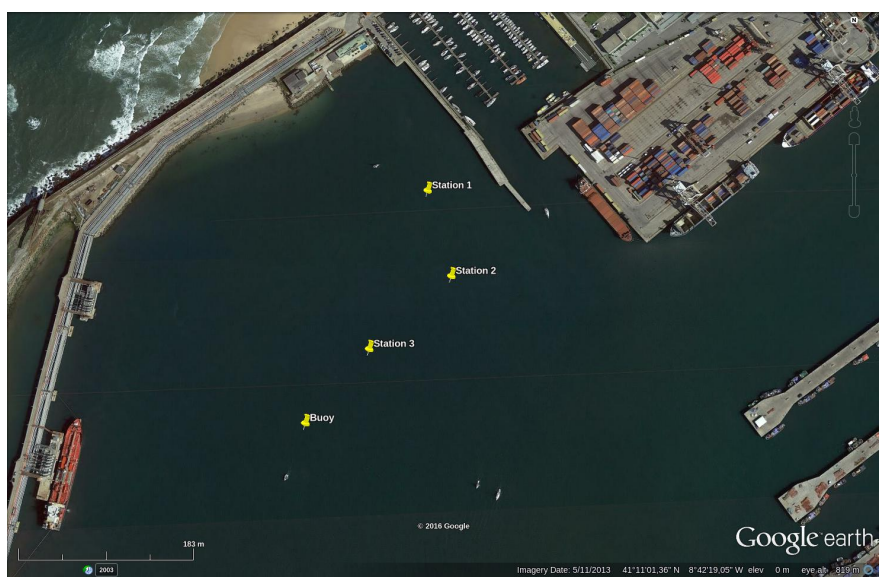

Fig. 5. Aerial view of area of operations in Leixões harbour, Porto, Portugal

The pinger was operated in two different modes. In mode one, it pings every second, synchronized with PPS signal, so that OWTT can be measured at the moored beacon. In the second mode, it works asynchronously, commanded by a PC to transmit every 3 seconds and waiting for a reply from the moored beacon, in order to measure TWTT. In this case, the acoustic transducer of the pinger was installed in the same pole as the GPS antenna, and lowered at about the same depth as the moored beacon, around $50 \mathrm{~cm}$.

The experimental procedure can then be summarized as follows:

1) Deploy the moored beacon and wait for the anchor to settle. Log all GPS positions throughout the mission;

2) Anchor the RHIB in station \#1, about $100 \mathrm{~m}$ away from the moored beacon, waiting for it to settle;

3) Start pinging the buoy with the pinger every second, synchronous with PPS;

4) Program the moored beacon with a reply map, so that it replies to a signal of frequency $f_{r}$ with a signal of frequency $f_{t}$ (this allows the pinger at the RHIB to measure TWTT);

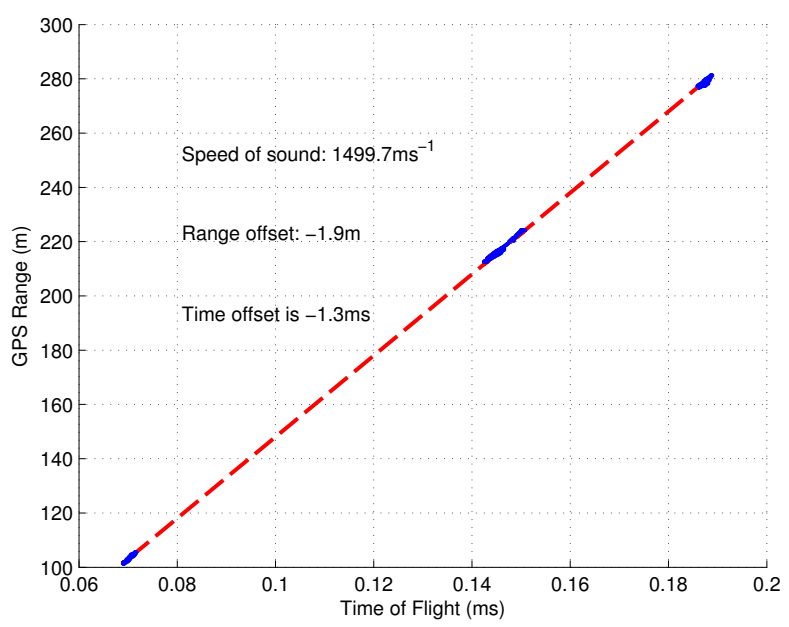

Fig. 6. Estimation of speed of sound, OWTT. In blue, experimental data collected, in red least-squares fit of the data.

5) Repeat steps 3 and 4 with the RHIB anchored at stations \#2 and \#3, located about 200 and 300 meters away from the moored beacon.

\section{EXPERIMENTAL VALIDATION}

One of the main objectives of the work here presented was to experimentally determine and characterize the sources of error, described previously, that can affect range measurements. In this section we will present those experimental results. In each station, data was collected for both the OWTT and TWTT situations, as described in the procedure, detailed in Section V. Besides acoustic data, we also collected GPS-data, which provided the necessary ground-truth data. Moreover, GPS data was also used to estimate the local speed of sound.

\section{A. OWTT}

The first step of the experimental validation is to estimate the speed of sound. To do so, we compared the GPS-ranges with the acoustic timing data for the three locations. Then we used a Least Square Estimator to fit the data. The result is plotted in Figure 6. There, it can be seen how the collected data, in blue, fits the linear model for the speed of sound, in red. The obtained estimate for the speed of sound was $1500 \mathrm{~ms}^{-1}$. This test also allowed us to estimate the delay $t_{f}$ introduced in the reception of the signals, which was determined to be of around $1.3 \mathrm{~ms}$.

Next, we used these values to determine the acoustic ranges between the moored beacon and the pinger, and compare it to the ranges obtained by GPS. The purpose was to understand how precise and accurate can the acoustically determined ranges be. Figures $7 \mathrm{a}, 7 \mathrm{~b}$ and $7 \mathrm{c}$ compare the obtained acoustic and GPS ranges for the three stations. It can be seen that the acoustic ranges are very similar with the GPS ones. Furthermore the differences in ranges were computed, and a figure of an RMS range difference always less than $0.6 \mathrm{~m}$ was obtained. Additionally, the data obtained in the three different 

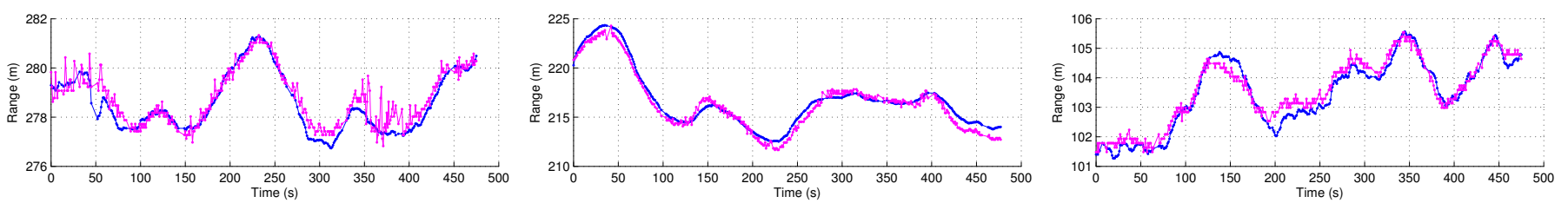

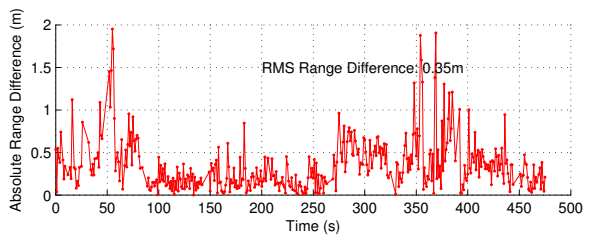

(a) Station 1

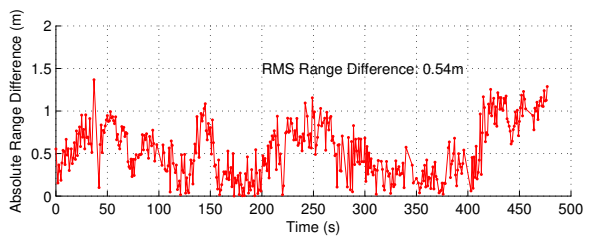

(b) Station 2

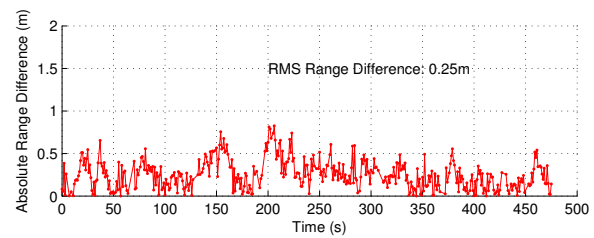

(c) Station 3

Fig. 7. Comparing GPS derived ranges with acoustic derived ranges, for the OWTT cases. In blue the GPS derived range and in magenta the acoustic derived range. The absolute error between the two sets of ranges can be seen in red.

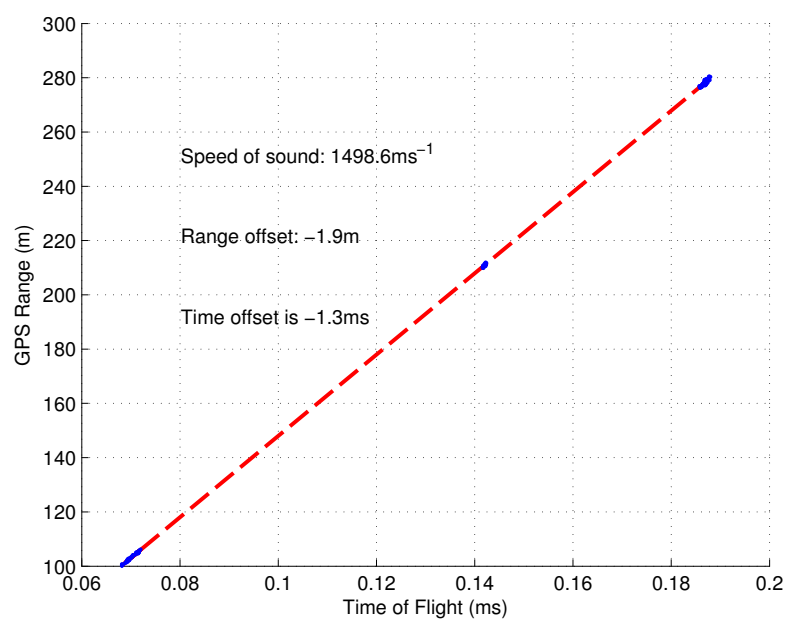

Fig. 8. Estimation of speed of sound, TWTT. In blue the experimental data collected, and the dashed lined the least-squares fit of the data.

stations provided evidence that the RMS range difference obtained is independent of range.

\section{B. TWTT}

For the TWTT case, a similar reasoning for the experimental characterization followed. Likewise, we first estimated the speed of sound, and the electronically induced delay detection, and used those values to compare ranges. Figure 8 illustrates how the collected data closely fits the linear model for the speed of sound. For the TWTT case the obtained value for the speed of sound was of $1499 \mathrm{~ms}^{-1}$ which differs from the value obtained before, for the OWTT, by less than $0.1 \%$. On the other hand, the detection delay was determined to be the same as the one for the OWTT case. This is in line with what was expected, as the delays introduced in both reception boards should be of similar level.

Following, we also compared the acoustic ranges with the ones obtained used GPS, displayed in Figure 9. In line with what was obtained before, also here the acoustic and GPS ranges are very similar, with RMS range differences always below $0.6 \mathrm{~m}$. This demonstrates the consistency of our results.

\section{Quantitative Analysis}

Despite the consistency of the results presented, the distribution of the differences in ranges between acoustic and GPS measurements was also analysed. The histogram of these differences, in Figure 10, can be of help to further understand the results. It can be seen that a large majority of the measurements falls within $0.4 \mathrm{~m}$. This is a clear indication that our system, with proper calibration, can in fact achieve performances similar to GPS. Figure 10 also shows the cumulative distribution of the differences in GPS and acoustic ranges. From there, we can establish that $60 \%$ the range measurements have less than $0.4 m$ difference to what was given by GPS, while $90 \%$ of all the measurements have a difference to GPS which falls within a $0.8 m$ difference.

\section{CONCLUSION}

This paper presented an experimental method to estimate sources of error that affect the accuracy of range measurements based on acoustics, as well as practical results obtained in field trials. They confirm that it is possible to estimate consistent delays in the acoustic detections, as well as accurate values for sound velocity. When these values are taken into account, the range estimates based on the time of flight of acoustic signals show an excellent agreement with ranges obtained using differences in GPS positions. Similar accuracies, with RMS differences below $0.6 \mathrm{~m}$, were obtained using OWTT and TWTT, demonstrating that there is no additional uncertainty caused by the replies in TWTT.

The precision of the range measurements obtained, as well as the excellent agreement with ranges obtained using GPS differences, indicate that these acoustic systems may be used in LBL navigation systems for AUVs, with similar performance as compared to GPS available at the surface. Moreover, this agreement also suggests that it may be possible to obtain an overall accuracy that even exceeds that of standard GPS. In order to assess this, a natural follow up of this work will be to 

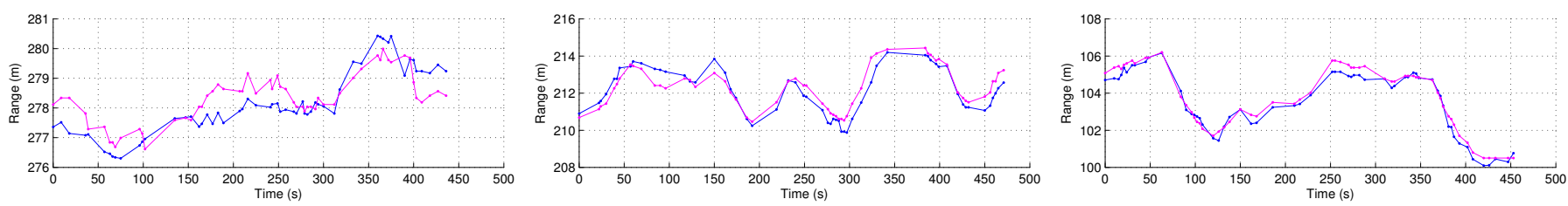

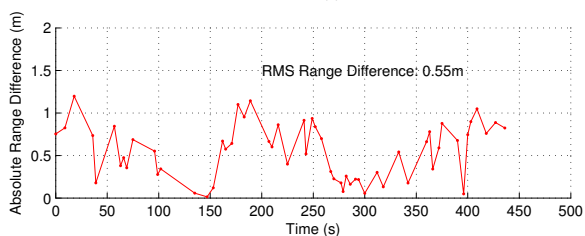

(a) Station 1

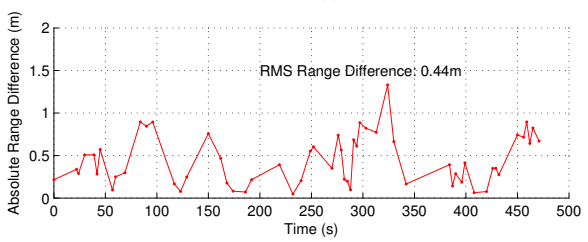

(b) Station 2

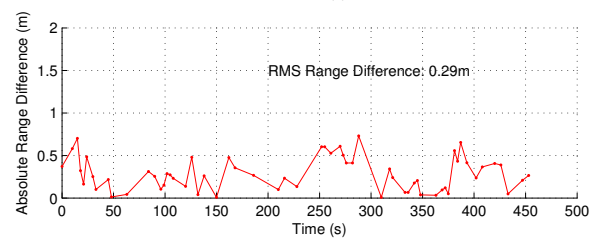

(c) Station 3

Fig. 9. Comparing GPS derived ranges with acoustic derived ranges, for the TWTT case. In blue the GPS derived range and in magenta the acoustic derived range. The absolute error between the two sets of ranges can be seen in red.

compare the results with differential GPS receivers installed in the acoustic pinger and receiver. Following this analysis of errors in point to point ranging, the next step will be to take advantage of the described procedure in future LBL navigation implementations in AUVs. In order to do it, it will be necessary to integrate a precise real time clock onboard the AUV, to ensure that the clock drift is negligible during the time the vehicle is submerged (i.e. without PPS). Throughout the AUV mission, the navigation beacons can also maintain a real time estimate of the sound velocity, either to transmit it to the AUV, or at least to be used for position corrections at the end of the mission.
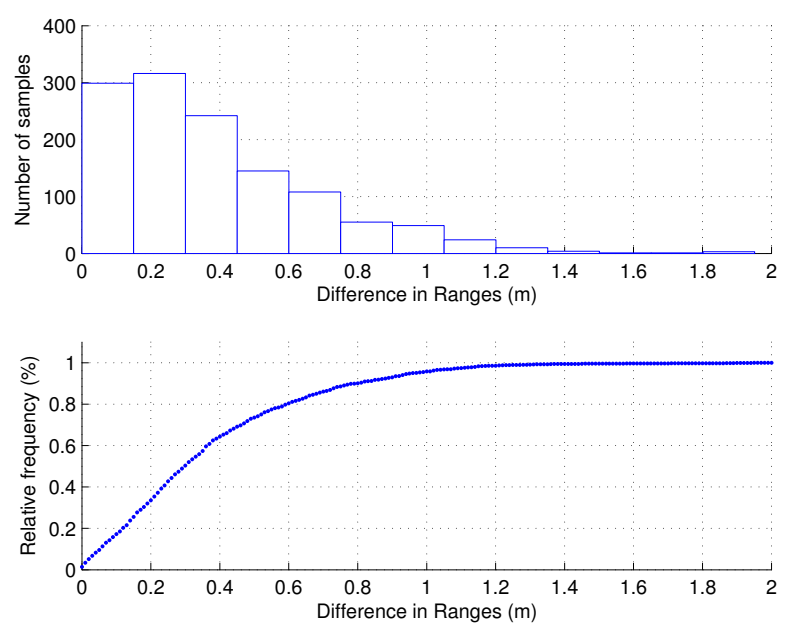

Fig. 10. Relative differences between GPS and given by acoustics.

\section{ACKNOWLEDGMENT}

This work is financed by the ERDF - European Regional Development Fund through the Operational Programme for
Competitiveness and Internationalisation - COMPETE 2020 Programme within project «POCI-01-0145-FEDER-006961», and by National Funds through the FCT - Fundação para a Ciência e a Tecnologia (Portuguese Foundation for Science and Technology) as part of project UID/EEA/50014/2013. The second author was supported by the Portuguese Foundation for Science and Technology through the Ph.D. grant SFRH/BD/70727/2010.

\section{REFERENCES}

[1] K. Vickery, "Acoustic positioning systems. a practical overview of current systems," in Autonomous Underwater Vehicles, 1998. AUV'98. Proceedings of the 1998 Workshop on, Aug 1998, pp. 5-17.

[2] L. Paull, S. Saeedi, M. Seto, and H. Li, "Auv navigation and localization: A review," IEEE Journal of Oceanic Engineering, vol. 39, no. 1, pp. 131149, Jan 2014.

[3] B. Bingham, "Predicting the navigation performance of underwater vehicles," in Proceedings of the 2009 IEEE/RSJ International Conference on Intelligent Robots and Systems, Piscataway, NJ, USA, Oct 2009, pp. 261-266.

[4] A. Turetta, G. Casalino, E. Simetti, A. Sperinde, and S. Torelli, "Analysis of the accuracy of a lbl-based underwater localization procedure," in Proc. of the IEEE/MTS Oceans'14 Conference, St. John's, Sept 2014, pp. 1-7.

[5] J. Melo and A. Matos, "Guidance and control of an asv in auv tracking operations," in Proc. of MTS/IEEE Oceans'08 Conference, Quebec, Canada, Sept 2008, pp. 1-7.

[6] R. M. Eustice, H. Singh, and L. L. Whitcomb, "Synchronous-clock, oneway-travel-time acoustic navigation for underwater vehicles," Journal of Field Robotics, vol. 28, no. 1, pp. 121-136, 2011.

[7] J. Melo and A. Matos, "A phd filter for tracking multiple auvs," in Proc. of MTS/IEEE Oceans'14 Conference, St. John's, Canada, Sept 2014, pp. $1-8$.

[8] _ , "Towards lbl positioning systems for multiple vehicles," in Proc. of MTS/IEEE Oceans'16 Conference, Shangai, China, April 2016, pp. 1-6.

[9] R. Almeida, N. Cruz, and A. Matos, "Man portable acoustic navigation buoys," in Proc. of MTS/IEEE Oceans'16 Conference, Shangai, China, April 2016, pp. 1-6. 